\title{
Design and Optimization of Micro Gas Sensor
}

\author{
Yu Guangbin ${ }^{1 *}$, Guan Yanqi ${ }^{1}$, Zhang Hongquan ${ }^{2}$ and Song Ye $^{1}$ \\ ${ }^{1}$ College of Mechanical Engineering, Harbin University of Science and \\ Technology, Harbin 150080, P.R. China \\ 2. School of Automation, Harbin Engineering University, Harbin150001, P.R. \\ China \\ yu_ccna@163.com
}

\begin{abstract}
The ordinary gas sensor has low material utilization, high power consumption, temperature distribution uneven and poor consistency problems, Based on film processing technology, this paper designs a new structure of micro oas sensor. Tiangle type design is first adopted in the structure, with platinum as the electrode material, ceramics substrate. Using ANSYS Workbench to analysis different size electrode and substrate that including the temperature field, stress field and the optimization design for substrate and electrode, the sensor can be uniform tempenature distribution, stress of small and the effective control of power consumption it is beneficial to improve the overall performance of the sensor. In addition, the sensor is tested to verify the accuracy of the finite element simulation.
\end{abstract}

Keywords: micro gas sensor: structure design; finite element analysis, performance testing

\section{Introduction}

The sensor is the fore-end and the main part of the information collection system. It is an indispensable link in the realization of modern measurement and automatic control, that is the source of the information. Its performance is directly related to the quality of the system data outside information perception[1-2]. So it is necessary to further research on the design and performance testing of the sensor.

For gas sensor generally it can be designed into contact with catalytic combustion type, solid electrolyte, electrochemical, optical and semiconductor sensor, etc[3-5]. The semiconductorgas sensor was divided into two types: resistance type and non-resistance type. From the point of view of design difficulty and practical value, semiconductor resistance type gas sensor has a wide range of measurement, high sensitivity, high cost performance, good stability, low power consumption, and it's easy to design and manufacture, so this kind of gas sensor has good prospects for development[6-7].

In recent years, with the development of design and production technology, the sensor also moving towards to precision, high performance and development of production integration, micro-structure gas sensor based on Micro Electronic System(MEMS) technology arise. Compared with the traditional gas sensor, the micro-structure gas sensor based on the Micro Electronic System (MEMS) technology integrates the heating electrode, the sensitive material and the measuring electrode into a whole. Compared with the traditional sensor that has advantages of low power consumption, good consistency, high degree of integration, and gradually becomes the main field of gas sensor structure[8-10]. Because the chemical activity of the sensitive materials generally in more than 280 degrees Celsius under the condition of high temperature can be excited, and uniform temperature distribution not only is conducive to the normal use of sensitive materials to play its characteristics, but also reduce power consumption and improve the 
response speed also has a positive impact, it is necessary to further design of microstructure gas sensor[11].

The substrate of the micro-structure gas sensors are mostly square, and the material is wasted phenomenon has always existed, simultaneously, the micro gas sensor also has the phenomenon of uneven temperature distribution field interference[12-13]. This paper designed a new type of structure for micro gas sensor, and using ANSYS Workbench on its temperature field and heat should be force are analyzed, to optimize the micro sensor electrode structure, the overall sensor high and uniform temperature distribution and reached the lowest power consumption [14-15].

\section{Structure Design and Optimization of Sensor}

The traditional gas sensor chip can make the 4 groups on the $4 * 4 \mathrm{~mm}$ substrates, however, the new structure of the sensor chip can make the 4 groups on the $3 * 3 \mathrm{~mm}$ substrates. A case study on the preparation of the sensor chip $12 * 12 \mathrm{~mm}$ ceramic) substrate, on the basis of the original increase of $33.3 \%$ of the utilization moregven a new type of triangular structure of the sensor chip has the measuring electrode the link of the measuring electrode is removed, as shown in Figure 1n

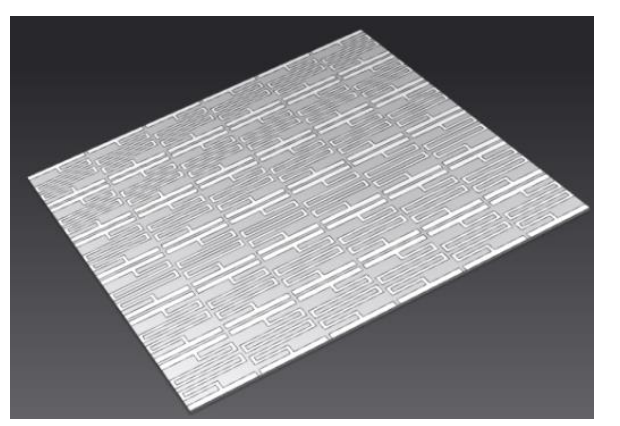

( a ) The Traditional Substrate Figure 1. Gas Sensor Chip Substrate

\subsection{Overall Design of the Sensor}

In this paper, a nery type of gas sensor with triangular structure is designed, which is shown in Figure 2 the plane size is $2.8 \times 1.98 \times 1.98 \mathrm{~mm}$, the bottom layer adopts ceramic material as substrate, in order to ensure the mechanical strength but also play a role in insulation, the reliability of the sensor is guaranteed. On the substrate, a snake shaped heating electrode and a measuring electrode are provided, the thickness is $2 \mathrm{um}$, and the heating electrode and the measuring electrode are designed in the same plane. The parasitic electric field accompanying of traditional sandwich structure sensor is avoided, but slso reduces the processing difficulty, it can be compatible with the existing plane integrated technology. Simultaneously, the novel type of triangular structure has further reduced the size of the sensor to make its contribution to the development of miniaturization. 


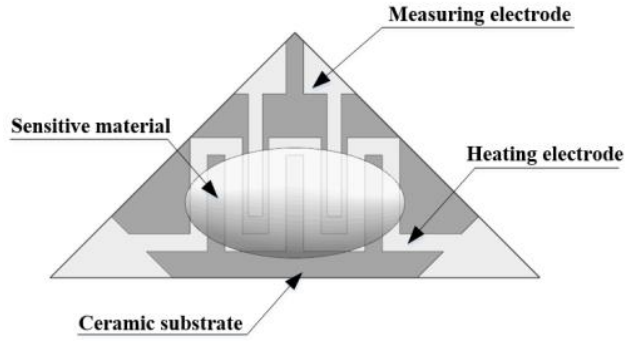

( a ) Sensor Top View

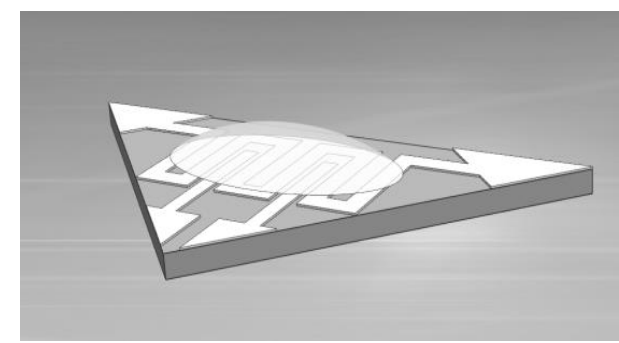

(b) Sensor 3D View

Figure 2. Schematic Diagram of Sensor Structure

\subsection{Optimization of Sensor Substrate}

Ceramic material is substrate, the oxygen ion is closed pack with six parties, ) and Al3+ is symmetrically distributed in an oxygen ion surrounded by eigh surface body coordination centers that lead to crystal lattice can be very large, therefore, it has the advantages of thermostability, low thermal resistance, corrosion resistance, high insulation and good heat loss prevention, the energy loss of the (senso 1 is reduced by using the characteristic of the ceramic substrate, in order to reduce the difficulty of cutting the substrate, and take into account the thermal uniformity as well as power consumption. There are two kinds of ceramic substrate with $50 \mathrm{um}$ and $100 \mathrm{um}$ thickness were designed, in order to compare the temperature field and thermal strain distribution of two kinds of ceramic substrate with different thickness, when the power consumption is $0.15 \mathrm{w}$, the heating electrode width of the senser is set to foo um, table 2-1 is the simulation material parameters, analysis of temperature field and thermal strain of two kinds for substrate with Workbench ANSYS, the results are shown in Figure 3; with the longest side triangular substrate corresponding to the vertex to the center point of the longest side to set the path, the temperature distribution of the path is obtained, as shown in Figure 4, through the analysis of the temperature field, the thermal stress distribution of the temperature fieldis shown in Figyre 5 .

\section{Table 2-1. Material Parameters}

\begin{tabular}{|c|c|c|c|c|c|c|c|}
\hline Material & $\begin{array}{l}\text { Thermal } \\
\text { conductivity } \\
\mathrm{W} /(\mathrm{m} \cdot \mathrm{k})\end{array}$ & $\begin{array}{l}\text { Specific } \\
\text { heat } \\
\mathrm{J} / \mathrm{KgK}\end{array}$ & $\begin{array}{l}\text { Density } \\
\mathrm{Kg} / \mathrm{m}^{3}\end{array}$ & $\begin{array}{l}\text { Thermal } \\
\text { expansion } \\
\text { coefficient } \\
/^{\circ} \mathrm{C}\end{array}$ & $\begin{array}{l}\text { Resistivity } \\
(300 \mathrm{~K} \cdot \Omega)\end{array}$ & $\begin{array}{l}\text { Elastic } \\
\text { modulus }\end{array}$ & $\begin{array}{l}\text { Poiss } \\
\text { on ratio }\end{array}$ \\
\hline Platinum & 71.4 & 133 & 21460 & 8.80E-06 & $1.06 \mathrm{E}-07$ & $6.20 \mathrm{E}+10$ & 0.39 \\
\hline Ceramic & 36 & 779 & 3890 & 7.7E-06 & $10 \mathrm{E}+06$ & $5.0 \mathrm{E}+07$ & 0.24 \\
\hline
\end{tabular}




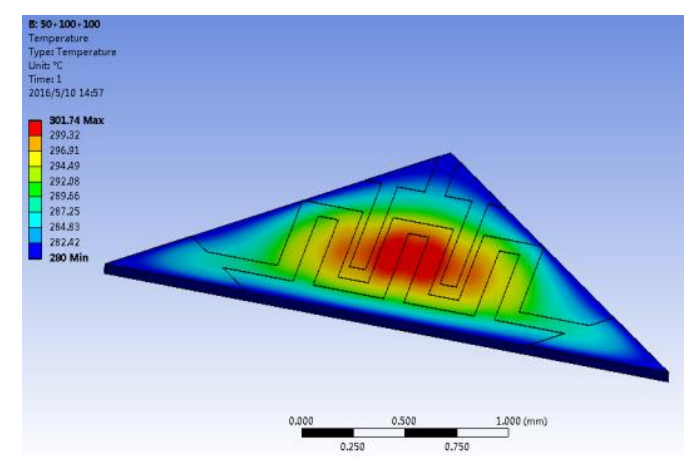

( a ) 50um Thick Substrate

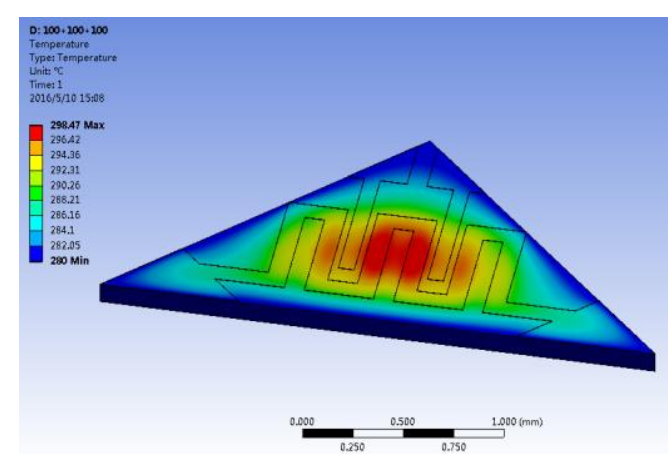

(b) 100um Thick Substrate

Figure 3. Temperature Field Distribution of Two Kinds of

\section{Substrate Thickness}

As shown in the Figure 3, the maximum temperature of the two kinds of substrates can be obtained in the central region, and the lowest temperature in the edge region. As can be seen from Figure 4, the temperature uniformity of the substrate with a thickness of 50um is lower than thickness of $100 \mathrm{um}$. The Figure 5 shovs that the maximum stress of the substrate with a thickness of 50um is at the corner of the heating electrode and is not uniform. The maximum stress of the thickpess with 100um is at the edge and is uniform,compare with each other the effect of the stress on the substrate is small. Under the condition of temperature that optimum working eondition of the sensor is substrate temperature uniformity and the thermalstress -1 s smanl, so the thickness of substrate is $100 \mathrm{um}$

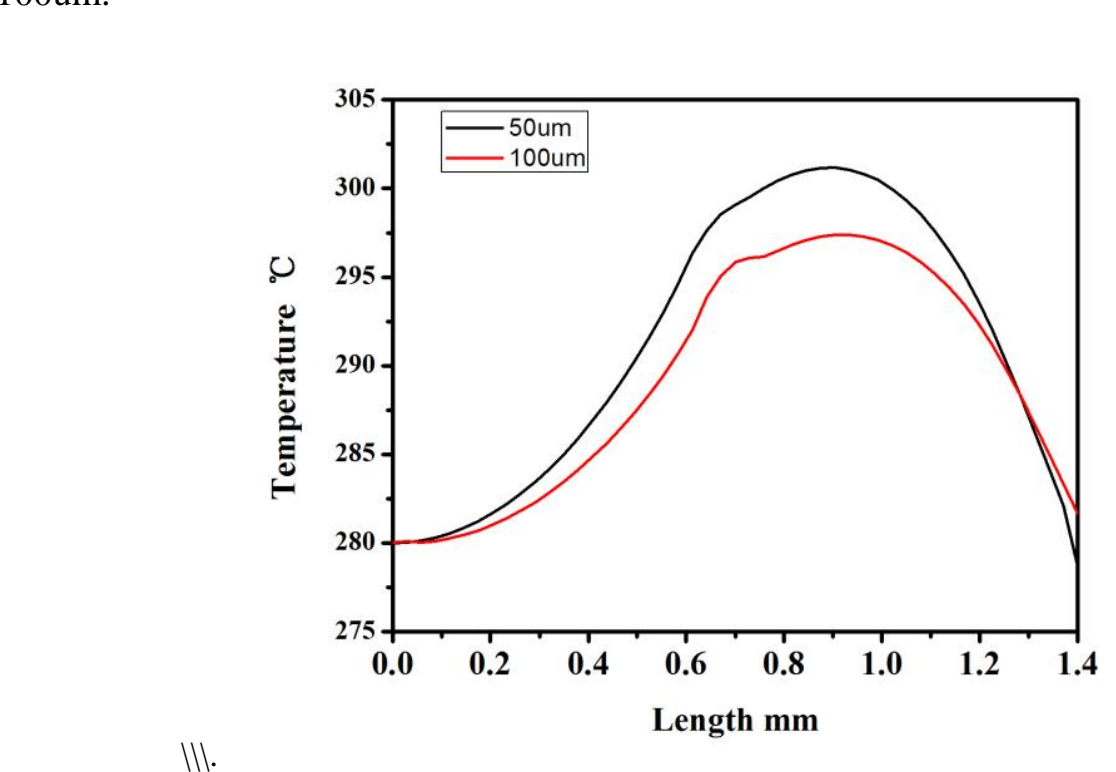

Figure 4. Temperature Distribution of the Same Path for Two Kinds of Substrates 


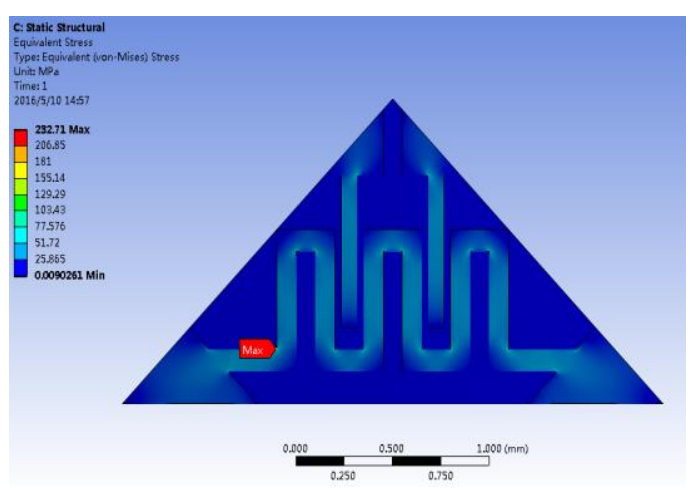

( a ) 50um Thick Substrate

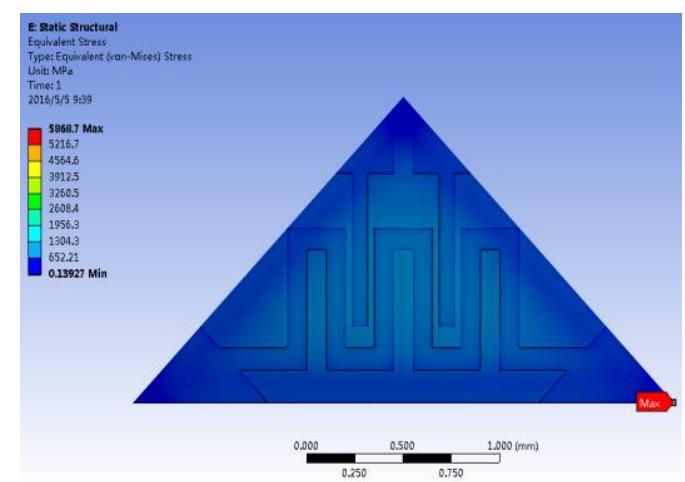

( b ) 100um Thick Substrate

Figure 5. Thermal Stress Distribution of Two Kinds of Substrates,

\subsection{Optimization of Heating Electrode}

When the heating electrode width is different that lead to the temperature and uniformity of the sensor have different effects. In order to find the best width of the heating electrode of the sensor, the thermal analysis of the heating electrode by Workbench ANSYS 15 to width of 50um and 100um under the power consumption of $0.15 \mathrm{w}$, and $100 \mathrm{um}$ is the maximum width of the heating electrode. At present, the thickness of the sensor substrate is 100un, and the measuring electrode width is 100um. The simulation results shown in Figure 6. when the of the heating electrode is 50um, the maximum temperature of the cenfral region is 288.62, and the temperature distribution is not uniform. When the heating electrode width is $100 \mathrm{um}$, the maximum temperature of the central region is 303.33 , and the temperature distribution is even better. So the heating electrode width of 100um has the relatively good temperature and uniformity

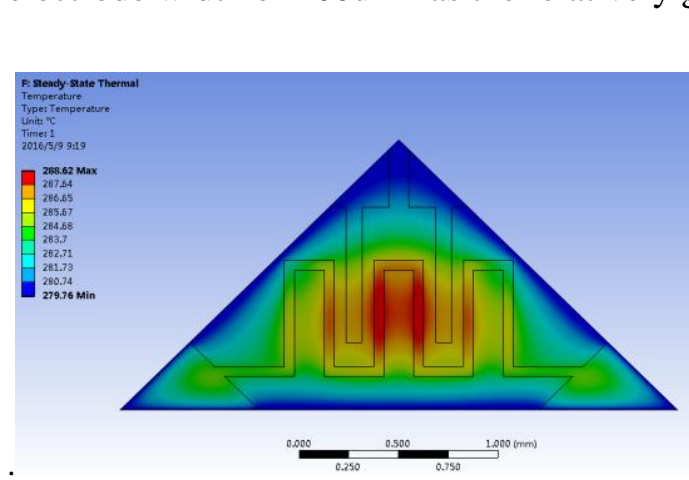

( a ) The Width is 50um

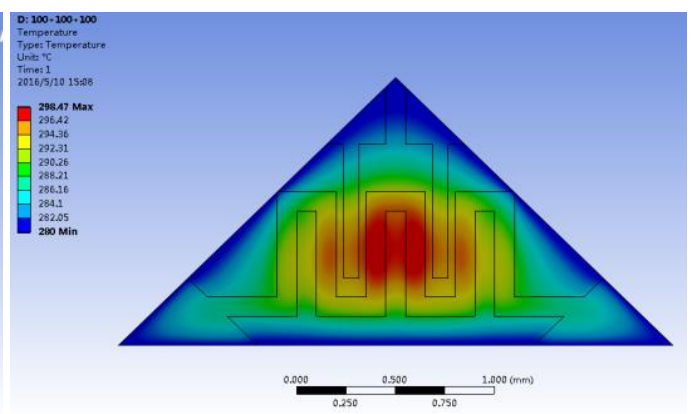

( b ) The $\mathrm{h}$ Width is 100um

\section{Figure 6. Temperature Field Distribution of Heating Electrode with Two Kinds of Width}

\subsection{Optimization of Measuring Electrode}

In the design of gas sensor, the temperature is affected by measuring electrode, in order to analyze the influence of measuring electrode for the temperature of sensor that electrode width is set to $50 \mathrm{um}$ and $100 \mathrm{um}$, in power for $0.15 \mathrm{w}$, the thickness of the substrate is 100um and the width of the heating electrode is $100 \mathrm{um}$ for the thermal analysis. The results as shown in Figure 7, from the figure can be seen the highest temperature of measuring electrode width is 100um higher than measuring electrode width 50um, and the same width with heating electrode then reduce the processing complexity, so the measuring electrode width of 100um. 


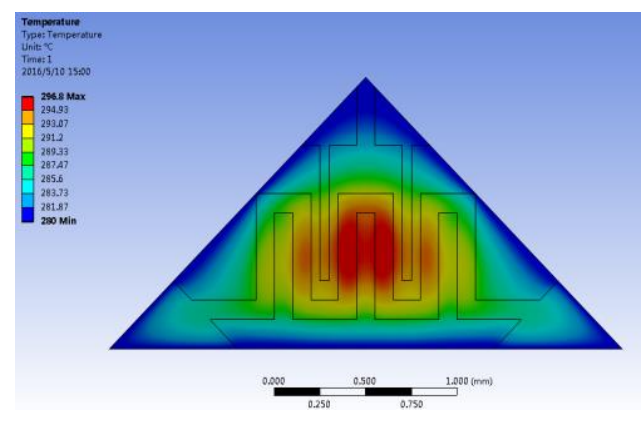

(a ) The width is 50um

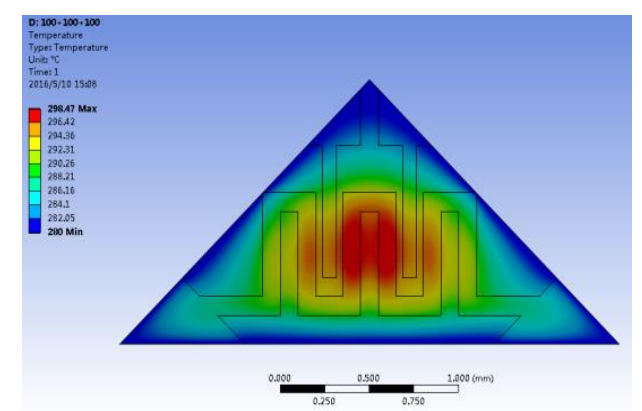

(b ) The width is 100um

Figure 7. Temperature Field Distribution of Measuring Electrode vith Dwo Kinds of Width

\section{Sensor Performance Test}

In this paper, a series of analysis for the new gas sensor, in odder to verify the reliability of the analysis, the following it will be based on the above analysis for the performance of the hydrogen sensor. The performance test or the sensor adopts a special dynamic gas testing device, which is used together with the standard gas dilution device, that can produce a volume fraction (0-10000) $\times 10^{6}$ standard hydrogen, the gas chromatographic detection of the final hy drogen concentrations is observed.

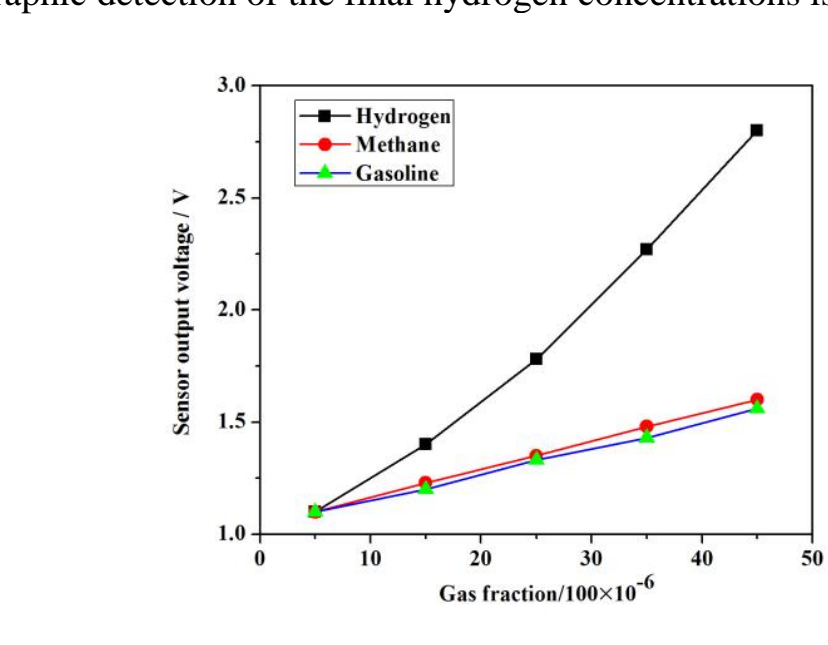

\section{Figure 8. Relationship between Sensor Output, Component Response to Hydrogen, Methane and Gasoline}

When the environment temperature is $22 \pm 0.5^{\circ} \mathrm{C}$, working voltage of $1.5 \mathrm{~V}$, humidity is $30 \pm 3 \% \mathrm{RH}$ and gas flow $150 \mathrm{ml} / \mathrm{min}$ of sensor for hydrogen, methane and gasoline corresponding voltage output as shown in Figure 8, the curve can be seen from the figure:sensor to the same concentration of hydrogen, methane and gasoline is an approximate linear relationship, hydrogen output voltage change rate is significantly higher than the other two gas ,it shows the sensor is more sensitive to hydrogen. 


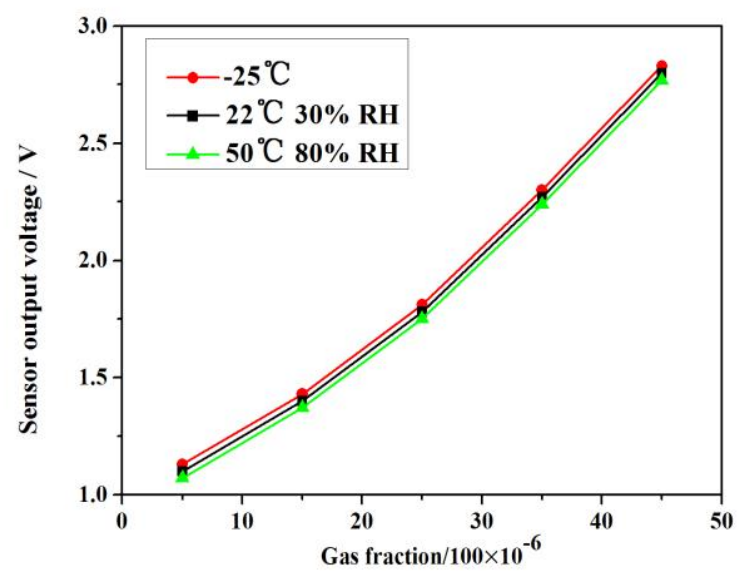

Figure 9. Relationship between the Sensor Output, Temperature and Humidity

In $50^{\circ} \mathrm{C} 80 \mathrm{RH} \%$ (high temperature and high humidity), $22^{\circ} \mathrm{C} 30 \mathrm{RH} \%$ (common temperature and common humidity), $-25^{\circ}$ (low temperature) conditions, we used ESPEC constant temperature humidity tes $\mathrm{b} \rho \mathrm{x}$ to simulate the above environment, the humidity uncertainty $* 2 \%$, the temperature uncertainty to $* 0.5 * \mathrm{C}$. The performance tests for the sensor that comparing the output data can be seen form Figure 9. From the test results, it can be known that the-measurement offset in extreme environment, compared to the normal use of the environment, the maximun output voltage fluctuation is less than $0.04 \mathrm{~V}$ and equivalent to a full range of $1.5 \% 0$.

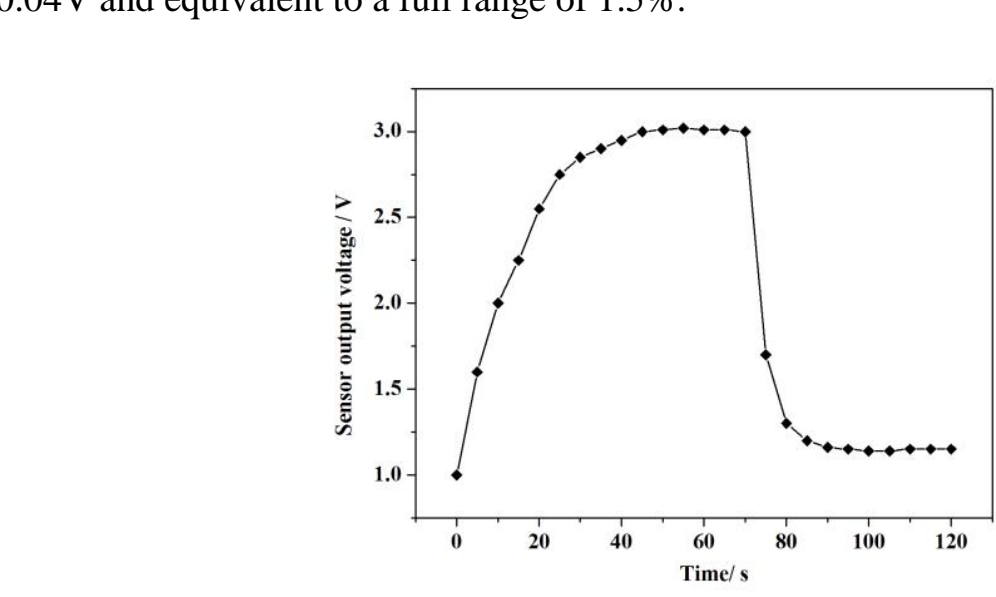

Figure 10. Sensor Response Curve and Recovery Time

We used special dynamic testing system to test the response to recovery time of the hydrogen sensor,and the output end of the sensor link data acquisition card by computer, and gas density using a $4000 \times 10^{-6}$ volume fraction, the results are as shown in Figure 10. According to the test results, we can observe that when the hydrogen sensor experiences a $2 \mathrm{~V}$ output, the recovery time is approximately $15 \mathrm{~s}$, the response to recovery time of the hydrogen sensor is standard. 


\section{Conclusions}

A new type of gas sensor with triangular structure is designed, the thickness of the substrate, the width of the heating electrode and the width of the measuring electrode that are analyzed and optimized by ANSYS Workbench, when the thickness of the substrate, the heating electrode and the measuring width are 100um, it brings about the sensor surface to obtain high and uniform temperature that is beneficial to improve the performance of the sensor. Finally, the performance of the sensor is tested, and the output voltage of the sensor is changed linearly then the test shows that the selectivity of the sensor is very good. It is compared with the traditional gas sensor that power consumption is $0.15 \mathrm{w}$, response to recovery time is short, the temperature and humidity of the interference are less than $1.5 \%$, and working stably. The design and application of the novel gas sensor have a certain reference value.

\section{Acknowledgment}

Special thanks are due to the Key Program of National Natural Serence Foundation of Heilongjiang under Grant (No.ZD201309), and supported by the Major Inernational Joint Research Program of China under Grant (No. 2014DFB 70120) and the National Natural Science Foundation of China (No. 51205093), Natural Science Important Fund Project in the Heilongjiang province (No.ZD201217977), and Creative Talent Special Project in Harbin (No.2010RFXXG009).

\section{References}

[1] Han N, Tian Y, Wu X, Improving humbidity selectivity in formaldehyde gas sensing by a two-sensor array made of Ga-doped ZnO.J Sensors and Actuators B. 138, 228 (2009)

[2] Pan Xianqing, Liu Qingcheng., Gas Sensor and its Development, J. Journal of East China Institute of Technology. 1, 89 (2004)

[3] Nagai, Daisuke ; Akannatsu, Takafuml\% Itoh, Toshio; Izu, Noriya; Shin, Woosuck Thermal balance analysis of a micro thermoelectric gas sensor using catalytic combustion of hydrogen, J. Sensors (Switzerland).14, 1822 (2014)

[4] Zhang Qiang, Guan'Zisheng Gas Sensors of Electric Resistance Semiconductors, J. Instrument Technique and Señsor. 7, 6 (2007)

[5] Liu Xin, Li Sbue, application and Development of Gas Sensors , J. Science and Technology of West China. 14, 13 (2008)

[6] Zhang Fengtian, Tang Zhenan, Gao Renjing, Jin Rencheng, Guo Wen-tai, Wang Haixia, Design and thermal analysis of gas pressure sensor with micro-hotplate, J. Optics and Precision Engineering. 6, 598 (2004)

[7] Blank, T. A., Eksperiandova, L. P., Belikov, K. N. Recent trends of ceramic humidity sensors development: A review, J. Sensors and Actuators B-Chemical.228, 416 (2016)

[8] Gupta, S.K., Joshi, Aditee; Manmeet, Kaur, Development of gas sensors using ZnO nanostructures, J. Jounnat of Chemical Sciences.122, 57 (2010)

[91 Hee.B. Yeo, C.H.; Shin, K.; Lee, K.J.; Lee, H.J.; Lee, W.B.; Ju, B.K, A micro-electromechanical system based hydrogen gas sensor, J. Sensor Letters. 6, 1014 (2008)

[10] Wang, Ping ; Zhang, Hong-Quan; Zhang, Tong; Wang, Bo-Ran; Wen, Dian-Zhong Research on micro twin-bridges catalytic combustion type kerosene gas sensor technology, J. Key Engineering Materials. 562-565,317 (2013)

[11] Yan Jun, Chen Xiangdong, Li Hui, Su Feng, Luo Xuesong ,Novel MEMS Gas Sensor and Its Theoretical Model . J. Design and Development of IC.33,435 (2008)

[12] Hui Chun,Xu Ailan,XuYulong, MEMS gas sensors. J. Journal of Functional Materials.2,133 (2003)

[13] Liu Lili, Wang Lianyuan, Guo Xin, Liu Zhen, Liu Li, Design and optimization of micro-hotplate electrode structure for micro gas sensor. J. Electronic Components and Materials. 33, 50 (2014)

[14] ZHANG Xiao-bo, SHAN Hao, LIU Li, WANG Lian-yuan, LI Shou-chun, WANG Guo-guang Design and optimization of structure of micro gas sensor. J. Transducer and Microsystem Technologies,30 . 128 (2013)

[15] Cho Nak-Jin, Lee Hyung-Kun, Moon Seung Eon, Stacked-type potentiometric solid-state $\mathrm{CO}_{2}$ gas sensor J. Sensors and Actuators, B: Chemical. 187, 340 (2013) 